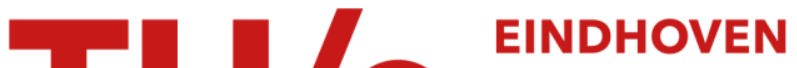 \\ UNIVERSITY OF \\ TECHNOLOGY
}

\section{Contour dynamics with symplectic time integration}

\section{Citation for published version (APA):}

Vosbeek, P. W. C., \& Mattheij, R. M. M. (1995). Contour dynamics with symplectic time integration. (RANA : reports on applied and numerical analysis; Vol. 9515). Technische Universiteit Eindhoven.

\section{Document status and date:}

Published: 01/01/1995

\section{Document Version:}

Publisher's PDF, also known as Version of Record (includes final page, issue and volume numbers)

\section{Please check the document version of this publication:}

- A submitted manuscript is the version of the article upon submission and before peer-review. There can be important differences between the submitted version and the official published version of record. People interested in the research are advised to contact the author for the final version of the publication, or visit the $\mathrm{DOI}$ to the publisher's website.

- The final author version and the galley proof are versions of the publication after peer review.

- The final published version features the final layout of the paper including the volume, issue and page numbers.

Link to publication

\section{General rights}

Copyright and moral rights for the publications made accessible in the public portal are retained by the authors and/or other copyright owners and it is a condition of accessing publications that users recognise and abide by the legal requirements associated with these rights.

- Users may download and print one copy of any publication from the public portal for the purpose of private study or research.

- You may not further distribute the material or use it for any profit-making activity or commercial gain

- You may freely distribute the URL identifying the publication in the public portal.

If the publication is distributed under the terms of Article $25 \mathrm{fa}$ of the Dutch Copyright Act, indicated by the "Taverne" license above, please follow below link for the End User Agreement:

www.tue.nl/taverne

Take down policy

If you believe that this document breaches copyright please contact us at:

openaccess@tue.nl

providing details and we will investigate your claim. 


\section{EINDHOVEN UNIVERSITY OF TECHNOLOGY}

Department of Mathematics and Computing Science

RANA 95-15

October 1995

Contour Dynamics with Symplectic

Time Integration

by

P.W.C. Vosbeek

R.M.M. Mattheij

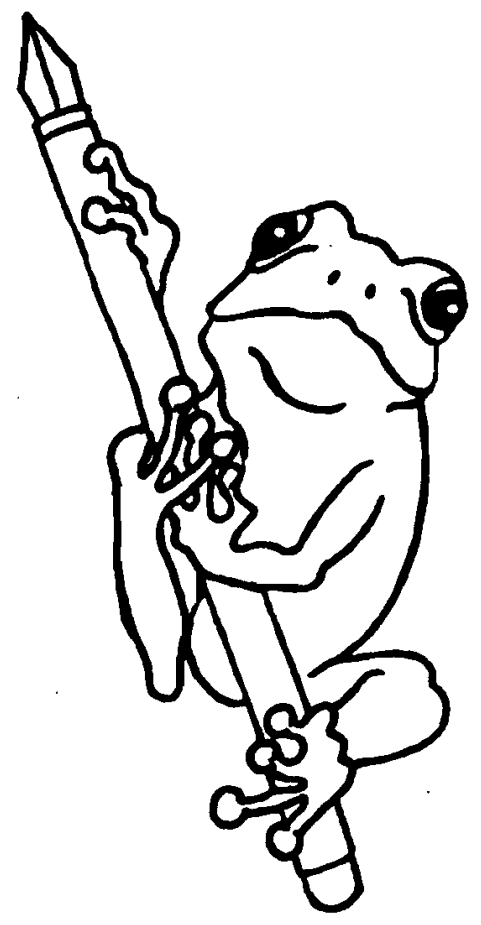


Reports on Applied and Numerical Analysis

Department of Mathematics and Computing Science Eindhoven University of Technology

P.O. Box 513

5600 MB Eindhoven

The Netherlands

ISSN: 0926-4507 


\title{
Contour Dynamics with Symplectic Time Integration
}

\author{
P.W.C. Vosbeek ${ }^{\dagger \ddagger}$, R.M.M. Mattheij ${ }^{\dagger}$ \\ $\dagger$ Department of Mathematics and Computing Science \\ $\ddagger$ Department of Physics \\ Eindhoven University of Technology \\ P.O. Box 513, $5600 \mathrm{MB}$ Eindhoven, The Netherlands \\ (e-mail: wsanpv@win.tue.nl,mattheij@win.tue.nl)
}

\begin{abstract}
In this paper we consider the time evolution of vortices simulated by the method of contour dynamics. Special attention is being paid to the Hamiltonian character of the governing equations and in particular to the conservational properties of numerical time integration for them. We assess symplectic and non-symplectic schemes. For the former methods, we give an implementation which is both efficient and yet effectively explicit. A number of numerical examples sustain the analysis and demonstrate the usefulness of the approach.
\end{abstract}

\section{Introduction}

In this paper we will discuss some aspects of the contour dynamics method, in particular the time integration. This well-known method is a useful tool for simulating vortices in twodimensional flows of an incompressible, inviscid fluid. The method and many improvements thereof, has been brought to full growth by the pioneering work of D.G. Dritschel ([1], [2]). Contour dynamics is based on the idea that the evolution of a patch of uniform vorticity is fully determined by the evolution of its boundary contour. The method is not limited to just one region of uniform vorticity; indeed, several contours can be nested in order to obtain an approximation of a patch of distributed vorticity (see [1], [2], [11]).

Two-dimensional flows of an incompressible, inviscid fluid can be described by Euler's equation, which expresses balance of linear momentum, and the continuity equation, which expresses conservation of mass. Regarding the latter conservation law we remark that, for an incompressible fluid, the velocity field is divergence free and thus, a stream function $\psi$ can be introduced in the usual way

$$
\left\{\begin{array}{l}
\dot{x}(t)=u(x, y, t)=\frac{\partial \psi(x, y, t)}{\partial y}, \\
\dot{y}(t)=v(x, y, t)=-\frac{\partial \psi(x, y, t)}{\partial x} .
\end{array}\right.
$$


Here $u$ is the component of the velocity field in $x$-direction and $v$ the component in $y$ direction. From this we can see that we are dealing with a Hamiltonian system with Hamiltonian $-\psi$. A very important property of such a system is the concept of preservation of area (see [9]) which in our case is equivalent to conservation of mass. Operators which have this property, are called symplectic. The solution operator of a Hamiltonian system is thus a symplectic operator. Since we like to solve this Hamiltonian system numerically, it is important, especially for long-time calculations, to preserve the area. This is possible if a so-called symplectic integration scheme is used (see [9]).

In contour dynamics, we are in fact dealing with two types of discretisations, viz. one in space and one in time. In this paper, we will show that the spatially discretised problem is also a Hamiltonian system, and therefore a symplectic time integration scheme is to be preferred to ordinary integration methods. Furthermore we shall outline how such a scheme can be applied to the the contour dynamics method and we show some results.

\section{The Governing Equations}

The vorticity vector $\boldsymbol{\omega}$ is defined as the curl of the velocity field $\mathbf{u}$. Since we consider a twodimensional flow in the $(x, y)$-plane, this vorticity vector points in a direction perpendicular to the $(x, y)$-plane; so we can write

$$
\boldsymbol{\omega}=\omega \mathbf{e}_{z} .
$$

By defining the stream function as in (1) and taking the curl of the linear momentum equation, we obtain the vorticity equation

$$
\frac{\partial \omega}{\partial t}+\frac{\partial \psi}{\partial y} \frac{\partial \omega}{\partial x}-\frac{\partial \psi}{\partial x} \frac{\partial \omega}{\partial y}=0
$$

which expresses conservation of vorticity of a fluid particle. Further, a relation between $\omega$ and $\psi$ can be derived from their definitions

$$
\nabla^{2} \psi=-\omega .
$$

By solving (3) using Green's function, we find an expression for $\psi$

$$
\psi(\mathbf{x}, t)=-\iint_{\mathbb{R}^{2}} G\left(\mathbf{x} ; \mathbf{x}^{\prime}\right) \omega\left(\mathbf{x}^{\prime}, t\right) d x^{\prime} d y^{\prime}, \quad t \geqslant 0,
$$

where $\mathbf{x}:=(x, y)^{T}$ and $G\left(\mathbf{x} ; \mathbf{x}^{\prime}\right):=\frac{1}{2 \pi} \ln \left\|\mathbf{x}-\mathbf{x}^{\prime}\right\|$ is Green's function in 2-D. The norm $\|\cdot\|$ is defined by $\|\mathbf{x}\|:=\sqrt{x^{2}+y^{2}}$, for each $\mathbf{x} \in \mathbb{R}^{2}$. Note that $\psi$ depends explicitly on time only if $\omega$ does; so if $\omega$ does not, then the equation is autonomous.

The initial continuous vorticity distribution $\omega$ is now approximated by a piecewise constant distribution $\tilde{\omega}$ like in Figure 1. By doing this, conservation of vorticity of a fluid 


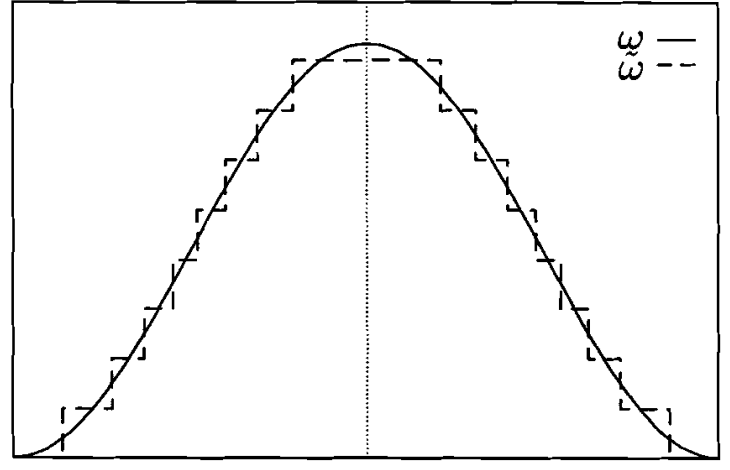

Figure 1: A cross-section of the continuous vorticity profile and the piecewise constant vorticity profile.

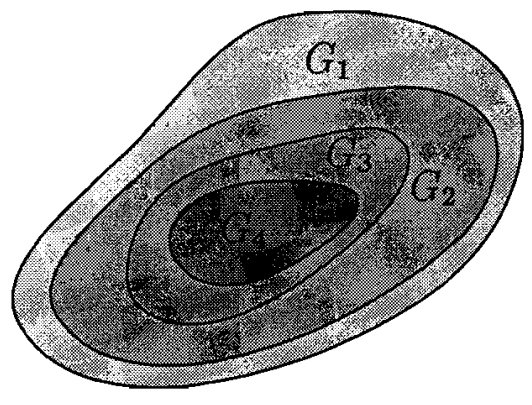

Figure 2: An arbitrary patch of piecewise constant vorticity distribution.

particle ensures that this distribution remains piecewise constant throughout time. Thus $\tilde{\omega}$ depends on time only through $\mathbf{x}$. For the approximate $\tilde{\psi}$ we then find

$$
\tilde{\psi}(\mathbf{x}):=-\sum_{m=1}^{M} \iint_{G_{m}} G\left(\mathbf{x} ; \mathbf{x}^{\prime}\right) \tilde{\omega}\left(\mathbf{x}^{\prime}\right) d x^{\prime} d y^{\prime}, \quad t \geqslant 0,
$$

where the $G_{m}$ are the regions of uniform vorticity (see e.g. Figure 2).

By applying Stokes' theorem for a scalar field, we can derive an expression for the velocity field:

$$
\tilde{\mathbf{u}}(\mathbf{x})=-\sum_{m=1}^{M} \omega_{m} \oint_{C_{m}(t)} G\left(\mathbf{x} ; \mathbf{x}^{\prime}\right) \mathbf{t} d s
$$

where $\omega_{m}$ is the jump of vorticity when crossing the contour $C_{m}(t)$ outward, and $\mathrm{t}$ is the unit vector tangential to the contour. Note that this system of equations is also Hamiltonian, with Hamiltonian $-\tilde{\psi}$.

\section{Spatial Discretisation}

\subsection{Discretisation of the Contours}

From (4), we see that the velocity $\tilde{\mathbf{u}}$ at any point of the two-dimensional plane is determined by a sum of contour integrals. To calculate these contour integrals numerically, we discretise each contour $C_{m}$ into a finite but adjustable number of nodes. Between two adjacent nodes, 
the contours are approximated by so-called elements. These elements can e.g. be linear (in this case, the two adjacent nodes are simply connected by a straight line segment), or quadratic, cubic, etc. The parameterisation $\mathbf{x}_{n}(\xi)$ of an element $e_{n}$ with nodes $\mathbf{x}_{n}$ and $\mathbf{x}_{n+1}$, is chosen such that $\mathbf{x}_{n}(-1)=\mathbf{x}_{n}, \mathbf{x}_{n}(1)=\mathbf{x}_{n+1}$ and $\mathbf{x}_{N}(1)=\mathbf{x}_{1}(-1)$. In the case of linear elements, this parameterisation is given by

$$
\mathbf{x}_{n}(\xi)=\frac{1}{2}(1-\xi) \mathbf{x}_{n}+\frac{1}{2}(1+\xi) \mathbf{x}_{n+1}
$$

The interpolated version of contour $C_{m}$ will be called $\hat{C}_{m}$.

The velocity $\hat{\mathbf{u}}:=(\hat{u}, \hat{v})^{T}$ at a point $\mathbf{x}$ anywhere in the flow field of the spatially discretised problem is then given by

$$
\begin{aligned}
\hat{\mathbf{u}}(\mathbf{x}) & =-\sum_{m=1}^{M} \frac{\omega_{m}}{2 \pi} \oint_{\hat{C}_{m}} \ln \left\|\mathbf{x}-\mathbf{x}^{\prime}\right\| \mathbf{t} d s \\
& =-\sum_{m=1}^{M} \frac{\omega_{m}}{2 \pi} \sum_{n=1}^{N} \int_{-1}^{1} \ln \left\|\mathbf{x}-\mathbf{x}_{n}(\xi)\right\| \dot{\mathbf{x}}_{n}(\xi) d \xi
\end{aligned}
$$

In all numerical examples, we shall use linear elements. The integrals in (6) along the elements can be determined using Gaussian quadrature. Only when $\mathbf{x}$ is equal to (or lying close to) one of the element nodes, an analytical solution of this integral is used; this is needed because the logarithm is (almost) singular in that case.

We will now prove the following property:

Property 3.1. The velocity field $\hat{\mathbf{u}}$ of the spatially discretised problem is divergence free.

Proof. The partial derivatives of the velocity field are given by

$$
\begin{aligned}
& \frac{\partial \hat{u}}{\partial x}=-\sum_{m=1}^{M} \frac{\omega_{m}}{2 \pi} \sum_{n=1}^{N} \int_{-1}^{1} \frac{\left(x-x_{n}(\xi)\right) \dot{x}_{n}(\xi)}{\left\|\mathbf{x}-\mathbf{x}_{n}(\xi)\right\|^{2}} d \xi \\
& \frac{\partial \hat{v}}{\partial y}=-\sum_{m=1}^{M} \frac{\omega_{m}}{2 \pi} \sum_{n=1}^{N} \int_{-1}^{1} \frac{\left(y-y_{n}(\xi)\right) \dot{y}_{n}(\xi)}{\left\|\mathbf{x}-\mathbf{x}_{n}(\xi)\right\|^{2}} d \xi
\end{aligned}
$$

So, for the divergence we find

$$
\begin{aligned}
(\nabla, \hat{\mathbf{u}}) & =\frac{\partial \hat{u}}{\partial x}+\frac{\partial \hat{v}}{\partial y} \\
& =-\sum_{m=1}^{M} \frac{\omega_{m}}{2 \pi} \sum_{n=1}^{N} \int_{-1}^{1} \frac{\left(\left(x-x_{n}(\xi)\right) \dot{x}_{n}(\xi)+\left(y-y_{n}(\xi)\right) \dot{y}_{n}(\xi)\right)}{\left\|\mathbf{x}-\mathbf{x}_{n}(\xi)\right\|^{2}} d \xi
\end{aligned}
$$




$$
\begin{aligned}
& =\sum_{m=1}^{M} \frac{\omega_{m}}{2 \pi} \sum_{n=1}^{N} \int_{-1}^{1} d \ln \left\|\mathbf{x}-\mathbf{x}_{n}(\xi)\right\| \\
& =\sum_{m=1}^{M} \frac{\omega_{m}}{2 \pi} \sum_{n=1}^{N}\left[\ln \left\|\mathbf{x}-\mathbf{x}_{n}(1)\right\|-\ln \left\|\mathbf{x}-\mathbf{x}_{n}(-1)\right\|\right] \\
& =0,
\end{aligned}
$$

since $\mathbf{x}_{n}(1)=\mathbf{x}_{n+1}(-1)$ for all $n, 1 \leqslant n<N$, and $\mathbf{x}_{N}(1)=\mathbf{x}_{1}(-1)$. This even holds when $\mathbf{x}$ is equal to one of the nodes on the contour.

From this theorem it follows that the spatially discretised problem also has a Hamiltonian. We can give an explicit expression for this Hamiltonian (in our case the stream function with a minus sign):

Property 3.2. The stream function of the velocity field $\hat{\mathbf{u}}$ is given by

$$
\hat{\psi}(\mathbf{x})=\sum_{m=1}^{M} \frac{\omega_{m}}{4 \pi} \sum_{n=1}^{N} \int_{-1}^{1}\left[f\left(\mathbf{x}, \mathbf{x}_{n}(\xi)\right) \dot{x}_{n}(\xi)+g\left(\mathbf{x}, \mathbf{x}_{n}(\xi)\right) \dot{y}_{n}(\xi)\right] d \xi
$$

where

$$
\begin{aligned}
f\left(\mathbf{x}, \mathbf{x}_{n}(\xi)\right) & :=\left(x-x_{n}(\xi)\right) \arctan \left(\frac{y-y_{n}(\xi)}{x-x_{n}(\xi)}\right)-\left(y-y_{n}(\xi)\right) \ln \left\|\mathbf{x}-\mathbf{x}_{n}(\xi)\right\| \\
g\left(\mathbf{x}, \mathbf{x}_{n}(\xi)\right) & :=-\left(y-y_{n}(\xi)\right) \arctan \left(\frac{x-x_{n}(\xi)}{y-y_{n}(\xi)}\right)+\left(x-x_{n}(\xi)\right) \ln \left\|\mathbf{x}-\mathbf{x}_{n}(\xi)\right\| .
\end{aligned}
$$

Proof. We have to prove that

$$
\begin{aligned}
\text { (i) } & \frac{\partial \hat{\psi}(\mathbf{x})}{\partial y}=\hat{u}(\mathbf{x}), \\
\text { (ii) } & \frac{\partial \hat{\psi}(\mathbf{x})}{\partial x}=-\hat{v}(\mathbf{x}) .
\end{aligned}
$$

From the partial derivatives of $f$ and $g$ we find the following properties:

$$
\begin{aligned}
& \left\{\begin{array}{l}
\nabla_{\mathbf{x}} f\left(\mathbf{x}, \mathbf{x}_{n}(\xi)\right)=-\nabla_{\mathbf{x}_{n}} f\left(\mathbf{x}, \mathbf{x}_{n}(\xi)\right) \\
\nabla_{\mathbf{x}} g\left(\mathbf{x}, \mathbf{x}_{n}(\xi)\right)=-\nabla_{\mathbf{x}_{n}} g\left(\mathbf{x}, \mathbf{x}_{n}(\xi)\right)
\end{array}\right. \\
& \frac{\partial f\left(\mathbf{x}, \mathbf{x}_{n}(\xi)\right)}{\partial y_{n}(\xi)}-\frac{\partial g\left(\mathbf{x}, \mathbf{x}_{n}(\xi)\right)}{\partial x_{n}(\xi)}=2 \ln \left\|\mathbf{x}-\mathbf{x}_{n}(\xi)\right\| .
\end{aligned}
$$


where $\nabla_{\mathbf{x}}$ denotes the gradient with respect to $\mathbf{x}$ and similarly $\nabla_{\mathbf{x}_{n}}$ for the gradient with respect to $\mathbf{x}_{n}(\xi)$. By taking the derivative of (7) with respect to $y$ and using $(*)$ we find

$$
\frac{\partial \hat{\psi}}{\partial y}=-\sum_{m=1}^{M} \frac{\omega_{m}}{4 \pi} \sum_{n=1}^{N} \int_{-1}^{1}\left[\frac{\partial f\left(\mathbf{x}, \mathbf{x}_{n}(\xi)\right)}{\partial y_{n}(\xi)} \dot{x}_{n}(\xi)+\frac{\partial g\left(\mathbf{x}, \mathbf{x}_{n}(\xi)\right)}{\partial y_{n}(\xi)} \dot{y}_{n}(\xi)\right] d \xi
$$

Now, we use $(* *)$ to obtain

$$
\begin{aligned}
\frac{\partial \hat{\psi}}{\partial y}= & -\sum_{m=1}^{M} \frac{\omega_{m}}{4 \pi} \sum_{n=1}^{N} \int_{-1}^{1}\left[\frac{\partial g\left(\mathbf{x}, \mathbf{x}_{n}(\xi)\right)}{\partial x_{n}(\xi)} \dot{x}_{n}(\xi)+\frac{\partial g\left(\mathbf{x}, \mathbf{x}_{n}(\xi)\right)}{\partial y_{n}(\xi)} \dot{y}_{n}(\xi)\right] d \xi \\
& -\sum_{m=1}^{M} \frac{\omega_{m}}{2 \pi} \sum_{n=1}^{N} \int_{-1}^{1} \ln \left\|\mathbf{x}-\mathbf{x}_{n}(\xi)\right\| \dot{x}_{n}(\xi) d \xi \\
= & \hat{u}(\mathbf{x})
\end{aligned}
$$

Here, we used similar reasoning as in property 3.1. The first part (i) of the proof is now completed. The second part can be proven in a similar way.

Thus, $\hat{\psi}$ is the stream function belonging to the velocity field $\hat{\mathbf{u}}$. Of course, an arbitrary constant may be added to this stream function.

If we use linear elements for the interpolation of the contours and use $h_{n}$ to denote the length of element $e_{n}$, we find the following property for the discretisation error:

Property 3.3. If $h_{m a x}$ is defined as the maximum of all $h_{n}$, then

$$
\|\tilde{\mathbf{u}}-\hat{\mathbf{u}}\|=\left\|\sum_{m=1}^{M} \frac{\omega}{2 \pi} \oint_{\hat{C}_{m}-C_{m}} \ln \right\| \mathbf{x}-\mathbf{x}^{\prime}\left\|\mathbf{t} d s^{\prime}\right\|=\mathcal{O}\left(h_{m a x}^{2}\right) .
$$

Proof. For the sake of simplicity, only the situation of one contour $C$ is considered. However, the more general case (with more than one contour) can be treated in the same way. We assume that the nodes $\mathbf{x}_{n}$ of contour $\hat{C}$ are lying on the exact contour $C$. Then we find

$$
\begin{aligned}
\tilde{\mathbf{u}}-\hat{\mathbf{u}} & =\frac{\omega}{2 \pi} \int_{\hat{C}-C} \ln \left\|\mathbf{x}-\mathbf{x}^{\prime}\right\| \mathbf{t} d s^{\prime} \\
& =\frac{\omega}{2 \pi} \sum_{n=1}^{N} \oint_{e_{n}-C_{n}} \ln \left\|\mathbf{x}-\mathbf{x}^{\prime}\right\| \mathbf{t} d s^{\prime},
\end{aligned}
$$

where $e_{n}$ is the straight line segment (with length $h_{n}$ ) connecting two adjacent nodes $\mathbf{x}_{n}$ and $\mathbf{x}_{n+1}$ and $C_{n}$ is the part of contour $C$ that connects these points also (see Figure 3 ). We now consider the contribution vector $\delta_{n}$ of one contour $e_{n}-C_{n}$ (i.e. the 'local geometrical 


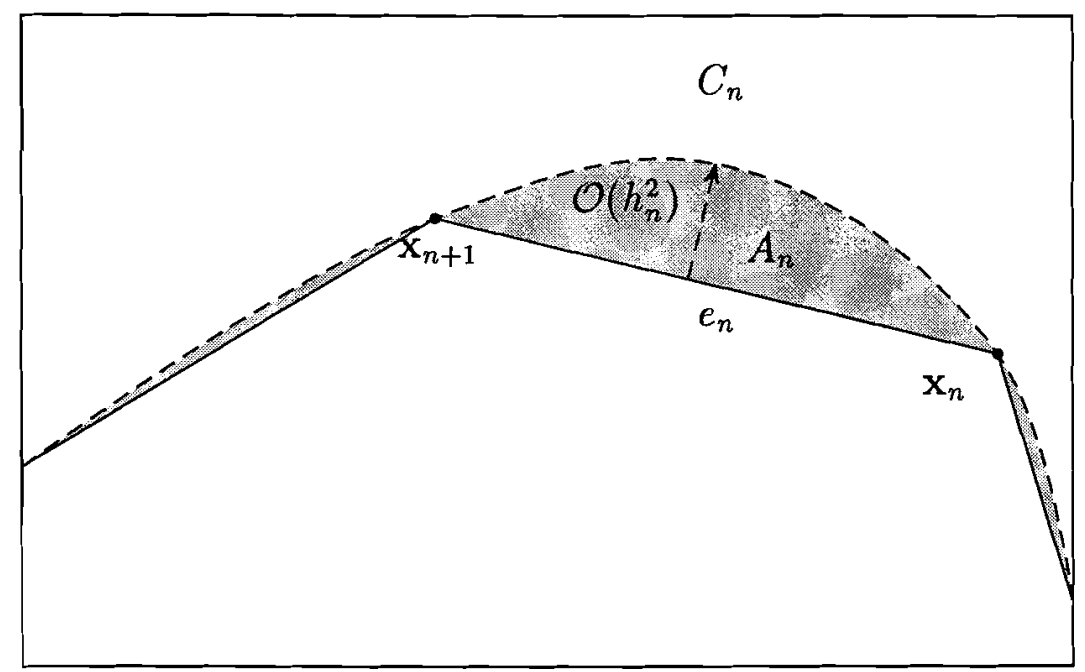

Figure 3: The region $A_{n}$ enclosed by $e_{n}$ and $C_{n}$.

error') to the discretisation error. We use Stokes' theorem for a vector field to obtain a surface integral over $A_{n}$ (see Figure 3 ) from the contour integral and find

$$
\begin{aligned}
\delta_{n}: & =\oint_{e_{n}-C_{n}} \ln \left\|\mathbf{x}-\mathbf{x}^{\prime}\right\| \mathbf{t} d s^{\prime} \\
& =\iint_{A_{n}} \mathbf{e}_{z} \times \nabla_{\mathbf{x}} \ln \left\|\mathbf{x}-\mathbf{x}^{\prime}\right\| d x^{\prime} d y^{\prime} \\
& =\iint_{A_{n}}\left(\frac{y-y^{\prime}}{\left\|\mathbf{x}-\mathbf{x}^{\prime}\right\|^{2}} \mathbf{e}_{x}-\frac{x-x^{\prime}}{\left\|\mathbf{x}-\mathbf{x}^{\prime}\right\|^{2}} \mathbf{e}_{y}\right) d x^{\prime} d y^{\prime} .
\end{aligned}
$$

We now introduce polar coordinates in the following way

$$
\begin{aligned}
& x-x^{\prime}:=r \cos (\varphi), \\
& y-y^{\prime}:=r \sin (\varphi) .
\end{aligned}
$$

Then, the surface integral becomes

$$
\delta_{n}=\int_{\varphi_{1}}^{\varphi_{2}} \int_{R_{1}(\varphi)}^{R_{2}(\varphi)}\left(\sin (\varphi) \mathbf{e}_{x}-\cos (\varphi) \mathbf{e}_{y}\right) d \varphi d r
$$

Here $\varphi_{1}, \varphi_{2}, R_{1}(\varphi)$ and $R_{2}(\varphi)$ are as in Figure 4. For the $x$-component we thus find

$$
\begin{aligned}
\left|\left(\boldsymbol{\delta}_{n}, \mathbf{e}_{x}\right)\right| & \leqslant \int_{\varphi_{1}}^{\varphi_{2}}\left|R_{2}(\varphi)-R_{1}(\varphi)\right||\sin (\varphi)| d \varphi \\
& \leqslant\left(\varphi_{2}-\varphi_{1}\right) \max _{\varphi_{1} \leqslant \varphi \leqslant \varphi_{2}}\left|R_{2}(\varphi)-R_{1}(\varphi)\right|
\end{aligned}
$$




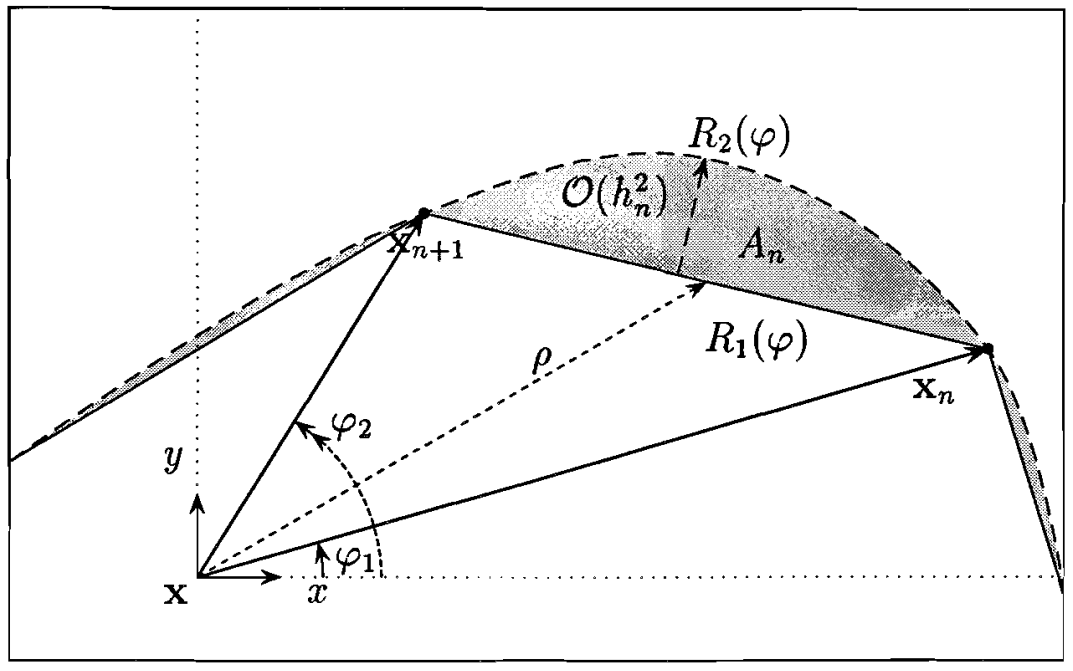

Figure 4: The situation of Figure 3 in polar coordinates.

and similar for the $y$-component

$$
\begin{aligned}
\left|\left(\delta_{n}, \mathbf{e}_{y}\right)\right| & \leqslant \int_{\varphi_{1}}^{\varphi_{2}}\left|R_{2}(\varphi)-R_{1}(\varphi)\right||\cos (\varphi)| d \varphi \\
& \leqslant\left(\varphi_{2}-\varphi_{1}\right) \max _{\varphi_{1} \leqslant \varphi \leqslant \varphi_{2}}\left|R_{2}(\varphi)-R_{1}(\varphi)\right|
\end{aligned}
$$

Because of the linear interpolation, we have $\left|R_{2}(\varphi)-R_{1}(\varphi)\right| \leqslant C h_{n}^{2}$ for $\varphi_{1} \leqslant \varphi \leqslant \varphi_{2}$.

Further, we can derive an expression for the angle $\vartheta:=\varphi_{2}-\varphi_{1}$. If we define $\rho$ by $\rho:=\left\|\mathbf{x}-\frac{1}{2}\left(\mathbf{x}_{n}+\mathbf{x}_{n+1}\right)\right\|$, then

$$
\begin{aligned}
\left\|\mathrm{x}-\mathrm{x}_{n}\right\|^{2} & =\rho^{2}+h_{n} \rho \cos \left(\vartheta_{1}\right)+\frac{1}{4} h_{n}^{2}, \\
\left\|\mathrm{x}-\mathrm{x}_{n+1}\right\|^{2} & =\rho^{2}-h_{n} \rho \cos \left(\vartheta_{1}\right)+\frac{1}{4} h_{n}^{2},
\end{aligned}
$$

where $\theta_{1}$ is the angle between $\mathbf{x}-\frac{1}{2}\left(\mathbf{x}_{n}+\mathbf{x}_{n+1}\right)$ and $\frac{1}{2}\left(\mathbf{x}_{n+1}-\mathbf{x}_{n}\right)$. For the inner product $\left(\mathbf{x}-\mathbf{x}_{n}, \mathbf{x}-\mathbf{x}_{n+1}\right)$ we simply have

$$
\left(\mathbf{x}-\mathbf{x}_{n}, \mathbf{x}-\mathbf{x}_{n+1}\right)=\rho^{2}-\frac{1}{4} h_{n}^{2}
$$

Since

$$
\cos (\vartheta)=\frac{\left(\mathbf{x}-\mathbf{x}_{n}, \mathbf{x}-\mathbf{x}_{n+1}\right)}{\left\|\mathrm{x}-\mathbf{x}_{n}\right\|\left\|\mathbf{x}-\mathbf{x}_{n+1}\right\|}
$$


we find after some calculation that

$$
\begin{aligned}
\sin ^{2}(\vartheta) & =1-\cos ^{2}(\vartheta) \\
& =\frac{\left(h_{n} / \rho\right)^{2} \sin ^{2}\left(\vartheta_{1}\right)}{1-\frac{1}{2}\left(h_{n} / \rho\right)^{2} \cos \left(2 \vartheta_{1}\right)+\frac{1}{16}\left(h_{n} / \rho\right)^{4}} .
\end{aligned}
$$

For $\rho>h_{n}$, we find from this that $\vartheta=\mathcal{O}\left(h_{n} / \rho\right)$.

Using this result in $(*)$ and $(* *)$, we have

$$
\left\|\delta_{n}\right\|=\left\{\begin{array}{l}
\mathcal{O}\left(h_{n}^{3} / \rho\right), \quad \text { if } \rho>h_{n}, \\
\mathcal{O}\left(h_{n}^{2}\right), \quad \text { if } \rho \leqslant h_{n} .
\end{array}\right.
$$

Now, by combining the contributions from all regions $A_{n}$, and by assuming that only few elements $e_{n}$ are lying close (i.e. closer then $h_{n}$ ) to the point $\mathbf{x}$, we may conclude that the overall discretisation error is $\mathcal{O}\left(h_{\text {max }}^{2}\right)$, where $h_{\text {max }}$ is the maximum element length on contour $\hat{C}$. This completes the proof.

Note that this discretisation error is not necessarily made every time step during the calculations. The first time this error is made, is when the initial contour grid is calculated. After that, such errors only occur when nodes are added or removed; if this is done properly the errors might be smaller.

\subsection{Node Distribution}

Since, in general, the shape of the contours becomes increasingly complex when time proceeds, the number of nodes initially placed on the contours, will not be enough to approximate the contours nicely at later time points. Therefore, the number of nodes on a contour may change during the calculations.

Several situations may occur where one has to add nodes to, or remove nodes from a contour. In general problems may arise when a small scale feature with a high density of nodes, encounters a larger scale feature with a lower node density (see Figure 5). In this case, the two parts of the contours may intersect, unless nodes are added properly to the large scale feature. To prevent this intersection of two (parts of) contours, the following is implemented: we say a node $\mathbf{x}_{i}$ is lying opposite to an element $e_{n}$ with nodes $\mathbf{x}_{n}$ and $\mathbf{x}_{n+1}$, if the line through $\mathbf{x}_{i}$, perpendicular to the line $l$ through $\mathbf{x}_{n}$ and $\mathbf{x}_{n+1}$ (see Figure 6) intersects $l$ in between $x_{n}$ and $x_{n+1}$. When no nodes are added between $x_{n}$ and $x_{n+1}$ and the local curvature at $\mathbf{x}_{i}$ is higher than the curvature at $\mathbf{x}_{n}$ or $\mathbf{x}_{n+1}$, such a point $\mathbf{x}_{i}$ may cause trouble. This can be avoided by properly adding a node between $\mathbf{x}_{n}$ and $\mathbf{x}_{n+1}$ each time the distance between the node and the element is becoming smaller than a given critical value. This distance is defined through the length of the vector $\mathbf{v}$ (see Figure 6 ). It is also possible that oppositely situated points do not cause trouble, and are even such that the local curvature is low enough to allow some nodes to be removed (for example on a filament); here, the local curvature is found from differentiation of a quadratic polynomial 


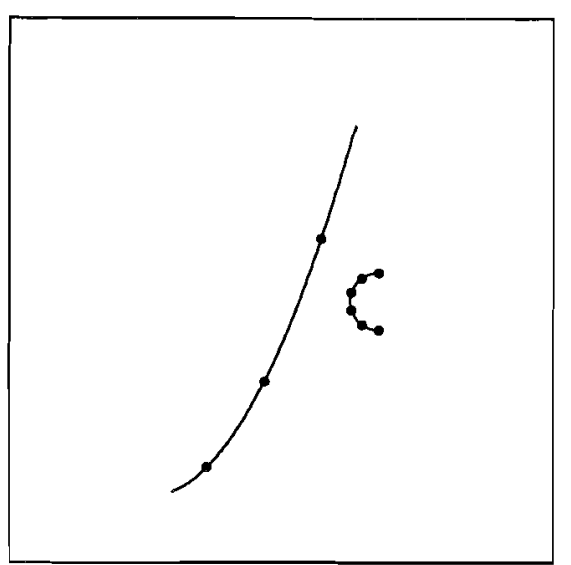

Figure 5: A small scale feature encountering a large scale one.

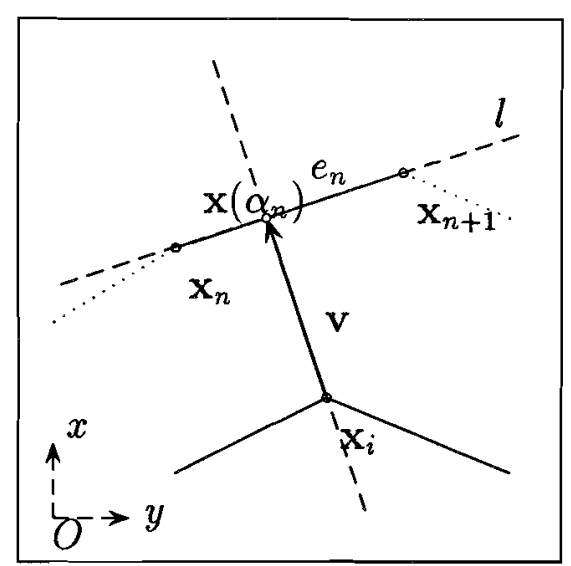

Figure 6: Node $\mathbf{x}_{i}$ is lying opposite to $e_{n}$.

through three consecutive points. Alternatively, nodes may have to be added at places where the local curvature is high in order to approximate the contour well enough. This can be formulated as follows: if $h_{n}$ is the length of element $e_{n}$, and $\kappa\left(\mathbf{x}_{n}\right)$ is the local curvature at node $\mathbf{x}_{n}$, then a node has to be added between $\mathbf{x}_{n}$ and $\mathbf{x}_{n+1}$ if

$$
\frac{1}{2}\left(\left|\kappa\left(\mathbf{x}_{n}\right)\right|+\left|\kappa\left(\mathbf{x}_{n+1}\right)\right|\right) h_{n}>\delta_{1},
$$

and node $\mathbf{x}_{n}$ has to be removed if

$$
\frac{1}{2}\left(\left|\kappa\left(\mathbf{x}_{n}\right)\right|+\left|\kappa\left(\mathbf{x}_{n+1}\right)\right|\right) h_{n}<\delta_{2}
$$

where $\delta_{1}$ and $\delta_{2}$ are given and $\delta_{1}>\delta_{2}$. Furthermore we require the element length to become no smaller than a minimum $h_{\min }$ and not larger than a maximum $h_{\max }$. The former requirement is made because the logarithm behaves badly for small arguments. The latter requirement is made to prevent the element length to become too large at filaments (where the curvature is very low at some places). The last condition the node distribution is required to satisfy, is that of quasi-uniformity. This can be formulated as

$$
\frac{h_{n-1}}{K} \leqslant h_{n} \leqslant K h_{n-1},
$$

where $K$ is a constant sufficiently larger than 1. This ensures that the element length of two neighbouring elements is not changing too much.

All together, we have four criteria for adding nodes and three for removing. It is obvious that the actual removal of nodes is very simple. For adding a node however, one has to 
decide where the new node has to be placed. To this end, we fit a quadratic polynomial through three successive nodes and place the new node on this polynomial. The velocity at the new node is also determined by quadratic interpolation.

\section{Time Integration}

As we can see from (6), the velocity field $\hat{\mathbf{u}}$ depends on the position of every node on every contour. Let $\mathbf{X}(t)$ be the vector of $x$-coordinates of all nodes at a certain time $t$

$$
\mathbf{X}(t):=\left(x_{1}(t), x_{2}(t), \ldots, x_{N}(t)\right)^{T}
$$

and $\mathbf{Y}(t)$ the vector of $y$-coordinates of all nodes

$$
\mathbf{Y}(t):=\left(y_{1}(t), y_{2}(t), \ldots, y_{N}(t)\right)^{T} \text {. }
$$

Denote the velocity in $x$-direction at node $\mathbf{x}_{n}$ by $\hat{u}_{n}(\mathbf{X}, \mathbf{Y})\left(:=\hat{u}\left(\mathbf{x}_{n}\right)\right)$ and in $y$-direction by $\hat{v}_{n}(\mathbf{X}, \mathbf{Y})\left(:=\hat{v}\left(\mathbf{x}_{n}\right)\right)$. Furthermore, let $\mathbf{U}$ be the vector of velocities in $x$-direction

$$
\mathbf{U}(\mathbf{X}, \mathbf{Y}):=\left(\hat{u}_{1}(\mathbf{X}, \mathbf{Y}), \hat{u}_{2}(\mathbf{X}, \mathbf{Y}), \ldots, \hat{u}_{N}(\mathbf{X}, \mathbf{Y})\right)^{T}
$$

and $\mathbf{V}$ the vector of velocities in $\mathrm{y}$-direction

$$
\mathbf{V}(\mathbf{X}, \mathbf{Y}):=\left(\hat{v}_{1}(\mathbf{X}, \mathbf{Y}), \hat{v}_{2}(\mathbf{X}, \mathbf{Y}), \ldots, \hat{v}_{N}(\mathbf{X}, \mathbf{Y})\right)^{T}
$$

For the time evolution of the contours, we now have to solve the following initial value problem

$$
\left\{\begin{array}{l}
\left(\begin{array}{c}
\dot{\mathbf{X}} \\
\dot{\mathbf{Y}}
\end{array}\right)=\left(\begin{array}{l}
\mathbf{U}(\mathbf{X}, \mathbf{Y}) \\
\mathbf{V}(\mathbf{X}, \mathbf{Y})
\end{array}\right), \quad t>0 \\
\left(\begin{array}{c}
\mathbf{X}(0) \\
\mathbf{Y}(0)
\end{array}\right)=\left(\begin{array}{l}
\mathbf{X}_{0} \\
\mathbf{Y}_{0}
\end{array}\right)
\end{array}\right.
$$

\subsection{Symplectic and Non-Symplectic Runge Kutta Methods}

As we have pointed out in section 3.1, the spatially discretised problem, of which we want to know the time evolution, is Hamiltonian. This means that the solution operator is symplectic (see [9]). In a numerical time integration, this solution operator is replaced by an approximate one. If we wish the latter to retain the Hamiltonian character of the former, we should insist the approximate solution operator on being symplectic as well. However, most standard numerical integrators replace the solution operator by a non-symplectic mapping. This is illustrated by the following example. Consider the time evolution of a circular vortex patch (initial radius equal to $r(0)$ ) of uniform vorticity $\omega$. The velocity field 


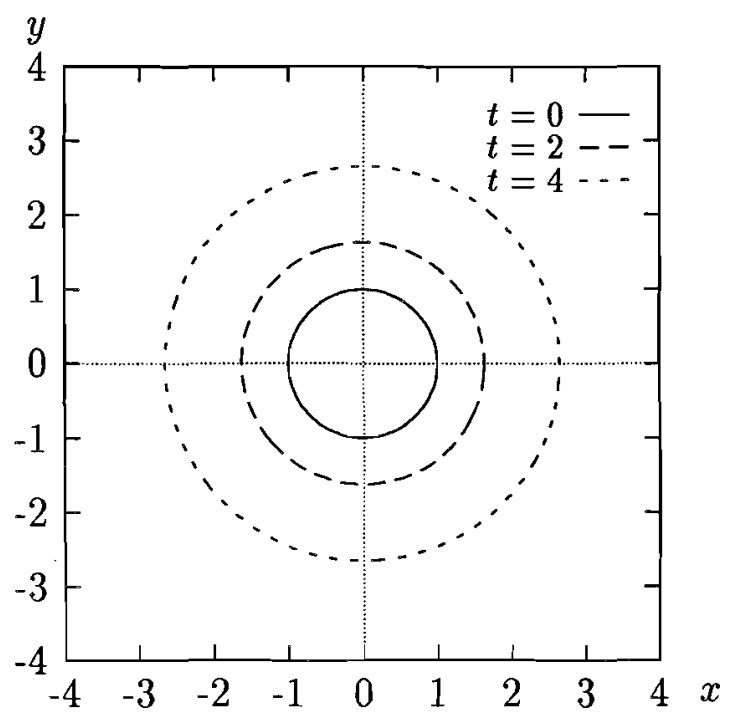

Figure 7: The evolution of a circular patch (initial radius equal to 1 ) of uniform vorticity $(\omega=2 \pi)$ using the Euler forward scheme with $\Delta t=0.05$.

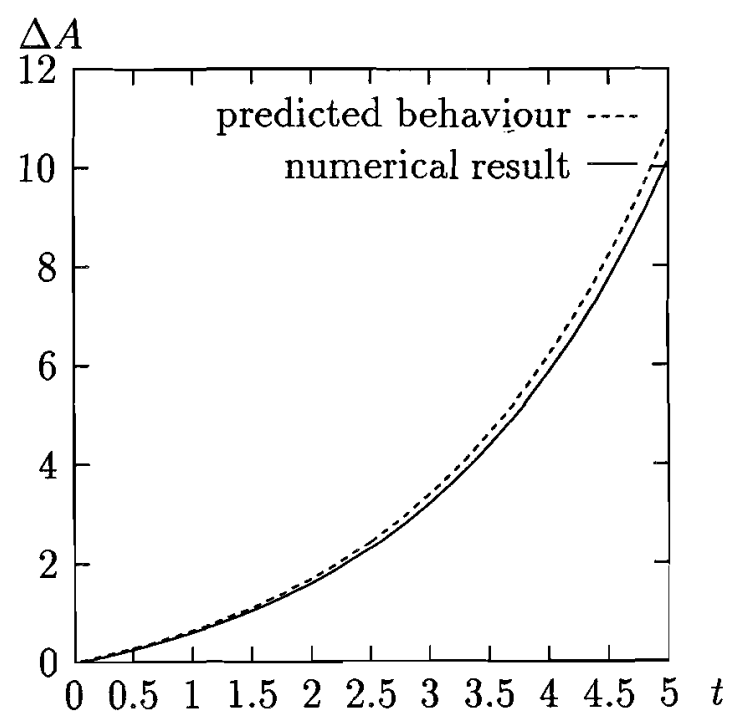

Figure 8: The predicted behaviour of $\Delta A$ and the numerically obtained $\triangle A$ as a function of time $t$. The area of the patch is calculated each time step using a contour integral expression.

inside the patch and on its boundary, is clearly given by (see [6])

$$
\left\{\begin{array}{l}
u(\mathbf{x})=\dot{x}=-\frac{\omega}{2} y, \\
v(\mathbf{x})=\dot{y}=\frac{\omega}{2} x,
\end{array} \quad t>0, \quad\|\mathbf{x}\| \leqslant 1\right.
$$

By applying the Euler forward scheme to (9), we find

$$
\left(\begin{array}{l}
x(t) \\
y(t)
\end{array}\right)=\left(\begin{array}{cc}
1 & -\frac{\omega \Delta t}{2} \\
\frac{\omega \Delta t}{2} & 1
\end{array}\right)\left(\begin{array}{l}
x(t-\Delta t) \\
y(t-\Delta t)
\end{array}\right),
$$

where $\Delta t$ is the time step. For the length $r(t)$ of the vector $\mathbf{x}$ after $t / \Delta t$ time steps, we thus find

$$
\begin{aligned}
r^{2}(t) & =x^{2}(t)+y^{2}(t) \\
& =\left(1+\left(\frac{\omega \Delta t}{2}\right)^{2}\right)\left(x^{2}(t-\Delta t)+y^{2}(t-\Delta t)\right)
\end{aligned}
$$




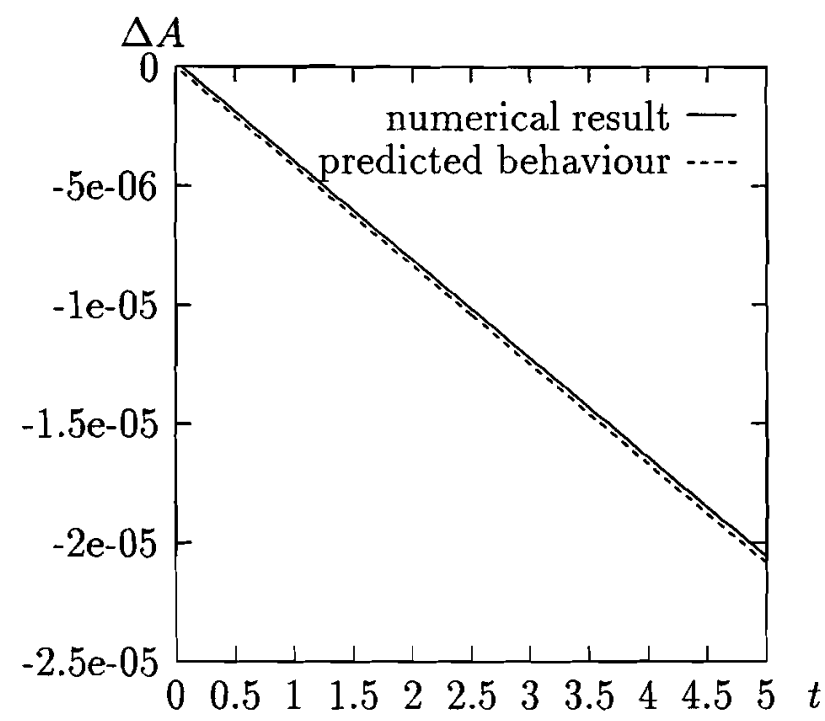

Figure 9: Similar as Figure 8 but for RK4 and $\Delta A:=(A(t)-A(0)) / A(0)$ (not the absolute value). Note the decrease of the area in this case.

$$
\begin{aligned}
& =\left(1+\left(\frac{\omega \Delta t}{2}\right)^{2}\right)^{t / \Delta t} r^{2}(0) \\
& =r^{2}(0) \exp \left(t \Delta t\left(\frac{\omega}{2}\right)^{2}+\mathcal{O}\left(t \Delta t^{3}\left(\frac{\omega}{2}\right)^{4}\right)\right) .
\end{aligned}
$$

The radius of the patch thus grows exponentially with time (see Figure 7 where we used $\omega=2 \pi$ and $\Delta t=0.05$ ). Since the patch remains circular for all time, we find for the area $A(t)$ of the patch after $t / \Delta t$ time steps

$$
\begin{aligned}
A(t) & =\pi r^{2}(t) \\
& =\pi r^{2}(0) \exp \left(t \Delta t\left(\frac{\omega}{2}\right)^{2}+\mathcal{O}\left(t \Delta t^{3}\left(\frac{\omega}{2}\right)^{4}\right)\right) .
\end{aligned}
$$

The interesting feature here is that

$$
A(t) \sim A(0) \exp \left(t \alpha^{2} \Delta t\right)
$$

where $\alpha=\omega / 2$; i.e. it becomes unbounded as $t \rightarrow \infty$, although it can be kept close to $A(0)$ on any finite interval by choosing $\Delta t$ small enough. In Figure 8 we have plotted $\Delta A:=|A(t)-A(0)| / A(0)$ versus time $t$ (where the area $\Delta A$ was calculated at each time step by a contour integral expression) and also the by (11) predicted behaviour of $\triangle A$. Clearly, (11) predicts the behaviour of the area very well. Further it may be clear that the Euler forward scheme is not symplectic and one may be forced to take many steps to stay close to conservation. In fact, this applies to any explicit method. Note that if we would apply the Euler backward scheme to this problem (although this would hardly be feasible 
in practice, since implementation would require the solution of a large linear system), we would find shrinking patches instead. For higher order (explicit) RK-schemes, a similar result applies, albeit with a more moderate growth. In particular, for the classical explicit fourth order RK-method (see [3], [4]), one finds by straightforward expansion

$$
A(t) \sim A(0) \exp \left(-t \alpha^{6} \Delta t^{5} / 72\right) .
$$

In Figure 9 we have plotted both the predicted and the numerically obtained values of $\triangle A$ as a function of time for the same problem as before (circular vortex patch). Here we omitted to take the absolute value of $(A(t)-A(0)) / A(0)$ in order to show the decrease of the area. Again, the results agree remarkably well with the theory. Although the variation of the area is much smaller in this case compared to the Euler forward scheme, it still can be of significant importance, for large time intervals or problems with larger values of $\alpha$.

Next, we will pay attention to a second order symplectic Runge Kutta (RK) scheme: the midpoint rule.

\subsection{Implementation of the Symplectic Midpoint Rule}

Symplectic RK-methods are implicit in general (see [9]). Therefore, the implementation of a symplectic method can be rather complex. If the problem would be stiff, Newtonlike iteration methods would be needed to solve the linear systems involved. In our case however, these systems are not stiff normally. Indeed, in order to follow the contour properly, we have to resolve regions which are moving relatively fast at all steps (probably at different places during the evolution), i.e. the 'smaller' time scales dictate the step size at any time. Therefore we can use a predictor-corrector scheme $\left(\mathbf{P}(\mathbf{E C})^{I} \mathbf{E}\right)$ for the implementation of the symplectic scheme. This means that the method will be explicit after all, but conservation can be achieved by choosing $I$ large enough. For this scheme, we shall choose the midpoint rule (which only is second order), but a similar implementation can be used for higher order symplectic methods.

Denote the vector of approximate $x$-coordinates of the positions of the nodes after $k$ time steps $\Delta t$ by $\mathbf{X}_{k}$ and the vector of $y$-coordinates by $\mathbf{Y}_{k}$. Furthermore, denote the vector of approximate velocities in $x$-direction by $\mathbf{U}\left(\mathbf{X}_{k}, \mathbf{Y}_{k}\right)$ and in $y$-direction by $\mathbf{V}\left(\mathbf{X}_{k}, \mathbf{Y}_{k}\right)$. Then the midpoint rule can be represented as

$$
\left(\begin{array}{l}
\mathbf{X}_{k+1} \\
\mathbf{Y}_{k+1}
\end{array}\right)=\left(\begin{array}{l}
\mathbf{X}_{k} \\
\mathbf{Y}_{k}
\end{array}\right)+\Delta t\left(\begin{array}{l}
\mathbf{U}\left(\frac{1}{2}\left(\mathbf{X}_{k}+\mathbf{X}_{k+1}\right), \frac{1}{2}\left(\mathbf{Y}_{k}+\mathbf{Y}_{k+1}\right)\right) \\
\mathbf{V}\left(\frac{1}{2}\left(\mathbf{X}_{k}+\mathbf{X}_{k+1}\right), \frac{1}{2}\left(\mathbf{Y}_{k}+\mathbf{Y}_{k+1}\right)\right)
\end{array}\right)
$$

or

$$
\left(\begin{array}{c}
\frac{1}{2}\left(\mathbf{X}_{k}+\mathbf{X}_{k+1}\right) \\
\frac{1}{2}\left(\mathbf{Y}_{k}+\mathbf{Y}_{k+1}\right)
\end{array}\right)=\left(\begin{array}{c}
\mathbf{X}_{k} \\
\mathbf{Y}_{k}
\end{array}\right)+\frac{1}{2} \Delta t\left(\begin{array}{c}
\mathbf{U}\left(\frac{1}{2}\left(\mathbf{X}_{k}+\mathbf{X}_{k+1}\right), \frac{1}{2}\left(\mathbf{Y}_{k}+\mathbf{Y}_{k+1}\right)\right) \\
\mathbf{V}\left(\frac{1}{2}\left(\mathbf{X}_{k}+\mathbf{X}_{k+1}\right), \frac{1}{2}\left(\mathbf{Y}_{k}+\mathbf{Y}_{k+1}\right)\right)
\end{array}\right)
$$

Introducing vector

$$
\overline{\mathbf{X}}:=\frac{1}{2}\left(\mathbf{X}_{k}+\mathbf{X}_{k+1}\right)
$$


and

$$
\overline{\mathbf{Y}}:=\frac{1}{2}\left(\mathbf{Y}_{k}+\mathbf{Y}_{k+1}\right)
$$

we apparently need to solve

$$
\left(\begin{array}{l}
\overline{\mathbf{X}} \\
\overline{\mathbf{Y}}
\end{array}\right)=\left(\begin{array}{l}
\mathbf{X}_{k} \\
\mathbf{Y}_{k}
\end{array}\right)+\frac{1}{2} \Delta t\left(\begin{array}{l}
\mathbf{U}(\overline{\mathbf{X}}, \overline{\mathbf{Y}}) \\
\mathbf{V}(\overline{\mathbf{X}}, \overline{\mathbf{Y}})
\end{array}\right)
$$

each time step. This is done by the following $\left(\mathbf{P}(\mathbf{E C})^{I} \mathbf{E}\right)$ method:

$$
\left.\begin{array}{ll}
\text { Predict: } & \left(\begin{array}{l}
\overline{\mathbf{X}}^{0} \\
\overline{\mathbf{Y}}^{0}
\end{array}\right)=\left(\begin{array}{l}
\mathbf{X}_{k} \\
\mathbf{Y}_{k}
\end{array}\right)+\frac{1}{2} \Delta t\left(\begin{array}{l}
\mathbf{U}\left(\mathbf{X}_{k}, \mathbf{Y}_{k}\right) \\
\mathbf{V}\left(\mathbf{X}_{k}, \mathbf{Y}_{k}\right)
\end{array}\right), \\
\text { Evaluate: } \quad \mathbf{U}\left(\overline{\mathbf{X}}^{i-1} \overline{\mathbf{Y}}^{i-1}\right) \text { and } \mathbf{V}\left(\overline{\mathbf{X}}^{i-1}, \overline{\mathbf{Y}}^{i-1}\right), \\
\text { Correct: } \quad\left(\begin{array}{l}
\overline{\mathbf{X}}^{i} \\
\overline{\mathbf{Y}}^{i}
\end{array}\right)=\left(\begin{array}{l}
\mathbf{X}_{k} \\
\mathbf{Y}_{k}
\end{array}\right)+\frac{1}{2} \Delta t\left(\begin{array}{c}
\mathbf{U}\left(\overline{\mathbf{X}}^{i-1}, \overline{\mathbf{Y}}^{i-1}\right) \\
\mathbf{V}\left(\overline{\mathbf{X}}^{i-1}, \overline{\mathbf{Y}}^{i-1}\right)
\end{array}\right),
\end{array}\right\} \quad \begin{gathered}
\text { for } i=1, \ldots, I \\
\text { Evaluate : } \quad \mathbf{U}\left(2 \overline{\mathbf{X}}^{I}-\mathbf{X}_{k}, 2 \overline{\mathbf{Y}}^{I}-\mathbf{Y}_{k}\right) \text { and } \mathbf{V}\left(2 \overline{\mathbf{X}}^{I}-\mathbf{X}_{k}, 2 \overline{\mathbf{Y}}^{I}-\mathbf{Y}_{k}\right) .
\end{gathered}
$$

The predictor step of this method is equivalent to the Euler forward scheme for obtaining a first approximation to $\mathbf{X}_{k+1}$ and $\mathbf{Y}_{k+1}$. At the last evaluation step (i.e. after $I$ cycles), the velocities at the (approximate) new positions

$$
\left(\begin{array}{l}
\mathbf{X}_{k+1} \\
\mathbf{Y}_{k+1}
\end{array}\right):=2\left(\begin{array}{l}
\overline{\mathbf{X}}^{I} \\
\overline{\mathbf{Y}}^{I}
\end{array}\right)-\left(\begin{array}{l}
\mathbf{X}_{k} \\
\mathbf{Y}_{k}
\end{array}\right)
$$

are calculated and used for the next time interval.

In general, the number of cycles to be performed, depends on both the order of the predictor scheme and the order of the corrector scheme. If the corrector is of order $p$ and the predictor of order $q(p \geqslant q)$, then the local discretisation error after $i$ cycles, $\delta^{(i)}$, is

$$
\delta^{(i)}=\mathcal{O}\left(\Delta t^{p}\right)+\mathcal{O}\left(\Delta t^{i+q}\right) \text {. }
$$

As far as standard accuracy arguments are concerned, we would not need to do more than $p-q+1$ cycles; so in our case, two cycles would be enough. However, a finite number of iterations implies an explicit integration after all and loss of its symplectic property. In order to obtain an (almost) symplectic scheme, we therefore have to do more cycles, basically till we have solved (13) within machine precision (see Figure 10). One should realise though, that each cycle requires the calculation of the velocity field, so this is very time consuming. To solve this problem, we use an extrapolation method to accelerate the convergence of the iteration process. The method we actually used is the so-called minimal polynomial extrapolation (MPE) method (see [10]). This MPE method is very suitable for our problem, since it is based on differences and does not need additional information of the Jacobian matrix. Before explaining the idea of this method, we will prove the following property: 


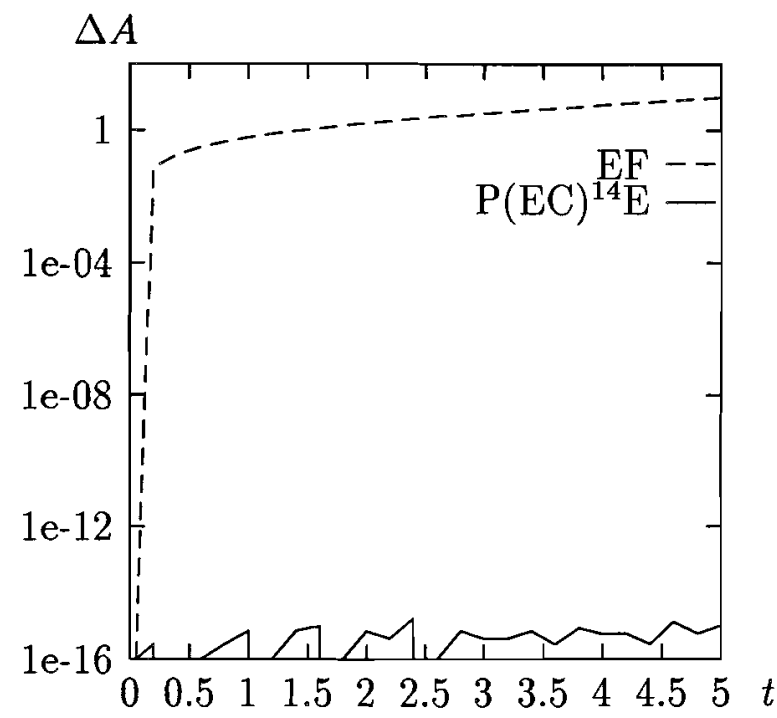

Figure 10: The same problem as in Figure 7; 14 cycles were needed to reach machine precision.

Property 4.1. For $i \geqslant 0$,

$$
\left(\begin{array}{l}
\overline{\mathbf{X}}^{i} \\
\overline{\mathbf{Y}}^{i}
\end{array}\right)=\left(\begin{array}{l}
\mathbf{X} \\
\overline{\mathbf{Y}}
\end{array}\right)-\left(\frac{1}{2} \Delta t\right)^{i+1} \mathbf{J}^{i}\left(\begin{array}{l}
\mathbf{U}(\overline{\mathbf{X}}, \overline{\mathbf{Y}}) \\
\mathbf{V}(\overline{\mathbf{X}}, \overline{\mathbf{Y}})
\end{array}\right)+\mathcal{O}\left(\Delta t^{i+2}\right),
$$

where $\mathbf{J}$ is the Jacobian matrix defined by

$$
\mathbf{J}:=\left(\begin{array}{ll}
\frac{\partial \mathbf{U}(\overline{\mathbf{X}}, \overline{\mathbf{Y}})}{\partial \overline{\mathbf{X}}} & \frac{\partial \mathbf{U}(\overline{\mathbf{X}}, \overline{\mathbf{Y}})}{\partial \mathbf{Y}} \\
\frac{\partial \mathbf{V}(\overline{\mathbf{X}}, \overline{\mathbf{Y}})}{\partial \overline{\mathbf{X}}} & \frac{\partial \mathbf{V}(\overline{\mathbf{X}}, \overline{\mathbf{Y}})}{\partial \overline{\mathbf{Y}}}
\end{array}\right) .
$$

Proof. The proof is by induction. For $i=0$, the result follows immediately from (13) since

$$
\left(\begin{array}{l}
\overline{\mathbf{X}}^{0} \\
\overline{\mathbf{Y}}^{0}
\end{array}\right)=\left(\begin{array}{l}
\mathbf{X}_{k} \\
\mathbf{Y}_{k}
\end{array}\right)=\left(\begin{array}{l}
\overline{\mathbf{X}} \\
\overline{\mathbf{Y}}
\end{array}\right)-\frac{1}{2} \Delta t\left(\begin{array}{l}
\mathbf{U}(\overline{\mathbf{X}}, \overline{\mathbf{Y}}) \\
\mathbf{V}(\overline{\mathbf{X}}, \overline{\mathbf{Y}})
\end{array}\right),
$$

Assume the property holds for $i$. Then we find with (13), the corrector part of the $\left(\mathbf{P}(\mathbf{E C})^{I} \mathbf{E}\right)$ scheme and a Taylor expansion of $\left(\mathbf{U}^{T}, \mathbf{V}^{T}\right)^{T}$ around $\left(\mathbf{X}^{T}, \mathbf{Y}^{T}\right)^{T}$

$$
\begin{aligned}
\left(\begin{array}{l}
\overline{\mathbf{X}}^{i+1} \\
\overline{\mathbf{Y}}^{i+1}
\end{array}\right) & =\left(\begin{array}{l}
\mathbf{X}_{k} \\
\mathbf{Y}_{k}
\end{array}\right)+\frac{1}{2} \Delta t\left(\begin{array}{l}
\mathbf{U}\left(\overline{\mathbf{X}}^{i}, \overline{\mathbf{Y}}^{i}\right) \\
\mathbf{V}\left(\overline{\mathbf{X}}^{i}, \overline{\mathbf{Y}}^{i}\right)
\end{array}\right) \\
& =\left(\begin{array}{l}
\mathbf{X}_{k} \\
\mathbf{Y}_{k}
\end{array}\right)+\frac{1}{2} \Delta t\left(\begin{array}{l}
\mathbf{U}(\overline{\mathbf{X}}, \overline{\mathbf{Y}}) \\
\mathbf{V}(\overline{\mathbf{X}}, \overline{\mathbf{Y}})
\end{array}\right)-\left(\frac{1}{2} \Delta t\right)^{i+2} \mathbf{J}^{i+1}\left(\begin{array}{l}
\mathbf{U}(\overline{\mathbf{X}}, \overline{\mathbf{Y}}) \\
\mathbf{V}(\overline{\mathbf{X}}, \overline{\mathbf{Y}})
\end{array}\right)+\mathcal{O}\left(\Delta t^{i+3}\right) \\
& =\left(\begin{array}{l}
\overline{\mathbf{X}} \\
\overline{\mathbf{Y}}
\end{array}\right)-\left(\frac{1}{2} \Delta t\right)^{i+2} \mathbf{J}^{i+1}\left(\begin{array}{l}
\mathbf{U}(\overline{\mathbf{X}}, \overline{\mathbf{Y}}) \\
\mathbf{V}(\overline{\mathbf{X}}, \overline{\mathbf{Y}})
\end{array}\right)+\mathcal{O}\left(\Delta t^{i+3}\right)
\end{aligned}
$$


This completes the proof.

From this property it follows that

$$
\begin{gathered}
\left(\begin{array}{l}
\overline{\mathbf{X}}^{i}-\overline{\mathbf{X}} \\
\overline{\mathbf{Y}}^{i}-\overline{\mathbf{Y}}
\end{array}\right)=\frac{1}{2} \Delta t \mathbf{J}\left(\frac{\overline{\mathbf{X}}^{i-1}-\overline{\mathbf{X}}}{\overline{\mathbf{Y}}^{i-1}-\overline{\mathbf{Y}}}\right)+\mathcal{O}\left(\Delta t^{i+2}\right), \quad \text { for } i \geqslant 1, \\
\left(\begin{array}{l}
\overline{\mathbf{X}}^{i}-\overline{\mathbf{X}}^{i-1} \\
\overline{\mathbf{Y}}^{i}-\overline{\mathbf{Y}}^{i-1}
\end{array}\right)=\frac{1}{2} \Delta t \mathbf{J}\left(\begin{array}{l}
\overline{\mathbf{X}}^{i-1}-\overline{\mathbf{X}}^{i-2} \\
\overline{\mathbf{Y}}^{i-1}-\overline{\mathbf{Y}}^{i-2}
\end{array}\right)+\mathcal{O}\left(\Delta t^{i+1}\right), \quad \text { for } i \geqslant 2,
\end{gathered}
$$

and the sequence $\left\{\overline{\mathbf{X}}^{i}\right\}$ is linearly convergent. Since MPE is based on differences, it will be convenient to have short notations for these. We define

$$
\mathbf{a}^{i}:=\left(\frac{\overline{\mathbf{X}}^{i}-\overline{\mathbf{X}}^{i-1}}{\overline{\mathbf{Y}}^{i}-\overline{\mathbf{Y}}^{i-1}}\right), \quad \text { for } 1 \leqslant i \leqslant I,
$$

and

$$
\mathbf{A}:=\left(\mathbf{a}^{0}, \mathbf{a}^{1}, \ldots, \mathbf{a}^{I-1}\right) .
$$

Now MPE calculates the fixed point $\left(\overline{\mathbf{X}}^{T}, \overline{\mathbf{Y}}^{T}\right)^{T}$ as a weighted average of the iterates with weights determined by the coefficients of the minimal polynomial $P(\lambda)$ of $\mathbf{J}$ with respect to $\mathbf{a}^{0}$. We take $I-1$ to be the degree $P(\lambda)$. Then, the minimal polynomial can be written as

$$
P(\lambda)=\sum_{i=0}^{I-1} c^{(i)} \lambda^{i}, \quad c^{(I-1)}=1 .
$$

Let the vector $\mathbf{c}:=\left(c^{(0)}, c^{(1)}, \ldots, c^{(I-2)}\right)^{T}$ be the vector of the unknown coefficients of the minimal polynomial. Then $\mathbf{c}$ is the solution of the system of equations

$$
\mathbf{A c}=-\mathbf{a}^{I-1} \text {. }
$$

In general, $I$ will be much smaller than the number of nodes. Thus, the system (17) has more equations than unknowns, but consistency can be proven (see [10]). Calculation of $\mathbf{c}$ requires only an LU-decomposition of $A$ and the solution of the upper triangular system, which is cheap compared to the calculation of the velocities. Once the vector $\mathbf{c}$ has been found, the fixed point can be calculated from

$$
\left(\sum_{i=0}^{I-1} c^{(i)}\right)(\overline{\mathbf{X}})=\sum_{i=0}^{I-1} c^{(i)}\left(\begin{array}{l}
\overline{\mathbf{X}}^{i+1} \\
\overline{\mathbf{Y}}^{i+1}
\end{array}\right) .
$$

Of course, we do not know the degree of the minimal polynomial. But this is not a problem in practice. If $I-1$ is larger than the degree of $P(\lambda)$, then there is no problem at 
all. If it is smaller, then instead of achieving equality in (17), the least squares solution gives coefficients of an 'almost annihilating' polynomial that is the 'best' monic polynomial of degree $I-1$ for eliminating the influence of $I-1$ dominant components of the error. These dominant components of the error are generated by the absolutely largest eigenvalues.

Consider now a vortex patch consisting of two concentric circular contours, where the outer contour rotates slower than the inner (the fluid enclosed by the inner contour has a larger uniform vorticity than the fluid enclosed by the outer and inner contour). Then the eigenvalues belonging to the outer contour are smaller (in absolute sense) than those of the inner one. So we might expect that, in the case where $I-1$ is smaller than the degree of the minimal polynomial, the area of the inner contour is better conserved than that of the outer contour. This is exactly what happens in Figure 11 and 12 . Here the evolution of a circular vortex patch with two contours has been calculated. The outer ring has vorticity $\omega=\pi$ and the inner circle has vorticity $\omega=2 \pi$. So the outer contour has eigenvalues which are smaller (in absolute value) than those of the inner. In Figure 12 we see that the area enclosed by the inner contour is better conserved than that of the outer, as expected. This suggests that it might be better to split up the extrapolation process over the contours: instead of calculating one set of coefficients $c^{(i)}$ for the whole system, a different set of coefficients is calculated for each contour in order to obtain the dominating terms for each contour. We have implemented this, and the results for the same vortex patch as in Figure 11 are shown in Figure results are much better and it turns out that not only the outer 13a and 13b. Indeed, the latter contour behaves better, but also the inner.

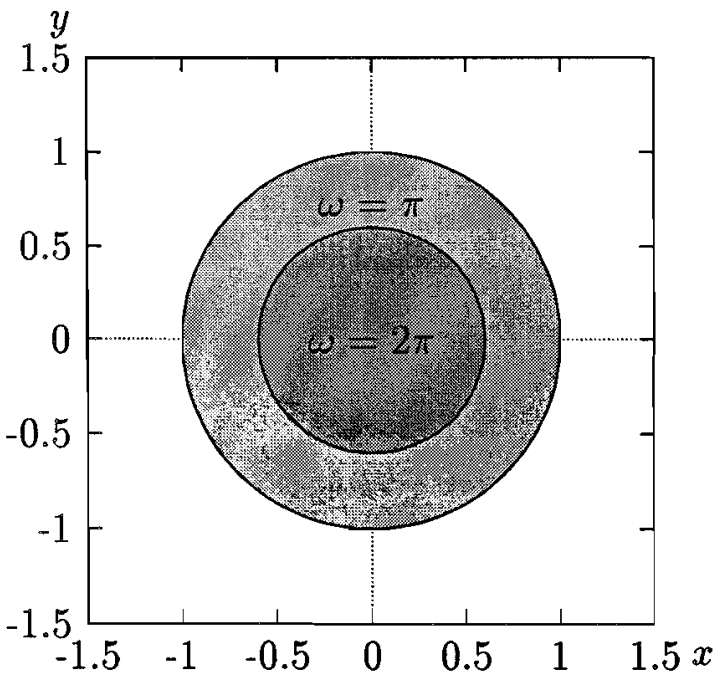

Figure 11: The situation at $t=0$ : the outer ring has vorticity $\omega=\pi$ and the region inside the inner circle has vorticity $\omega=2 \pi$.

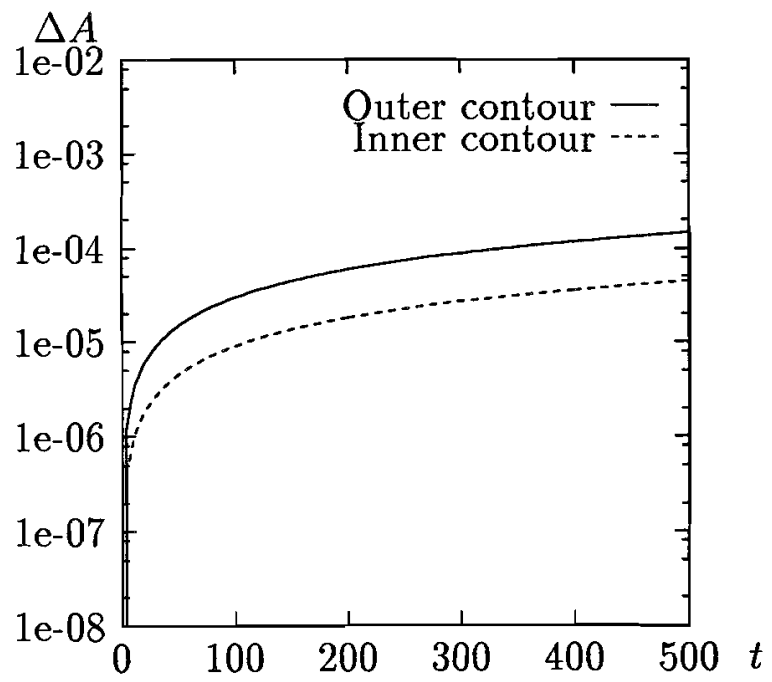

Figure 12: The variation $\Delta A$ of the area as a function of time $t$. The time step is taken to be $\Delta t=0.05$, the number of cycles is equal to 3 and MPE-extrapolation is performed. 


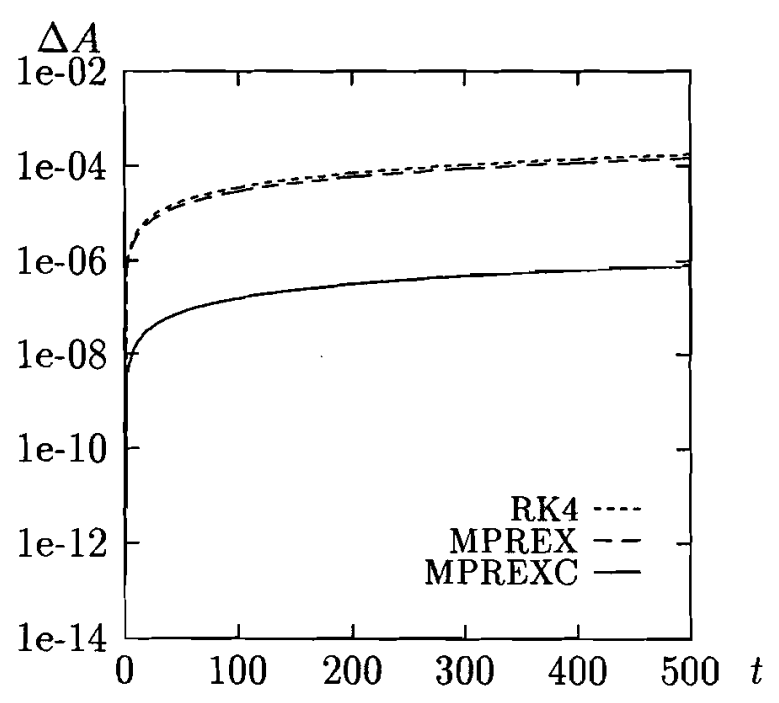

a) Contour 1

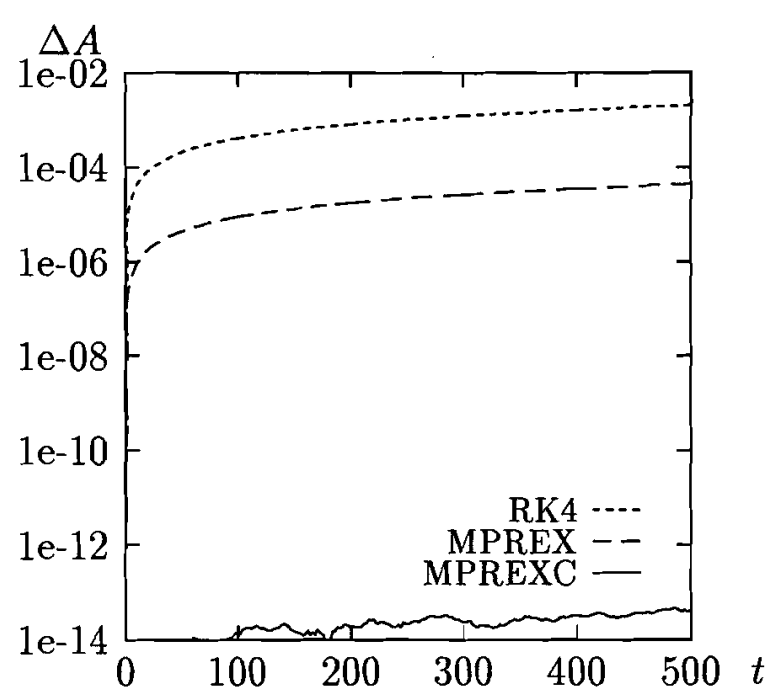

b) Contour 2

Figure 13: The variation of the area $\Delta A$ calculated with the midpoint rule with 3 cycles where the extrapolation part is splitted up over the contours (MPREXC), with the midpoint rule with 3 cycles with global extrapolation (MPREX) and with a fourth order RK-method (RK4). In all three cases, $\Delta t=0.05$.

This is due to the fact that larger $\omega$ makes the extrapolation more accurate (dominance of eigenvalue is more pronounced). In the next section we will show some more results. In Figure 13, also results are shown of the evolution of the same vortex patch but now with the classical fourth order explicit RK method. The results are in agreement with (12). It might be clear, that the results of the midpoint rule are much better than those of the RK method. This, while the same effort was needed for both methods: we used $I=3$, so the velocities had to be calculated four times per time step which is the same as for the fourth order RK method.

The other error, the phase shift, may also be investigated (although it is often less interesting in practical situations). A way to do this, is to follow the point on a contour which initially is placed at the positive part of the $x$-axis. The solution of the problem can be determined analytically, and we find that such a point of the outer contour passes the positive $x$-axis exactly 170 times and that of the inner 250 times. For all numerical

\begin{tabular}{||l|c|c|c|c||}
\hline & MPREXC & MPREX & RK4 & Exact \\
\hline \hline Contour 1 & 169.6 & 169.6 & 169.8 & 170 \\
\hline Contour 2 & 249.3 & 249.3 & 249.8 & 250 \\
\hline
\end{tabular}

Table 1: The number of revolutions of the outer and inner contour. 
methods, this turns out to be slightly less than these analytical values as can be seen in Table 1. However, the differences between the investigated methods are very small, so we may conclude that all numerical schemes produce a phase shift of comparable magnitude.

\section{$5 \quad$ Further Numerical Results}

In the previous section, we compared some numerical results obtained by the midpoint rule with a fourth order explicit RK method. However, the test problem was rather simple. Therefore, we will consider some more complex problems in this section. The first example is the evolution of a so-called Kida-vortex (see [5]). Initially, an elliptical patch, with aspect ratio equal to 0.75 and of uniform vorticity $\omega=2 \pi$, is placed in the centre of a strain flow $\mathbf{u}_{s}$, given by

$$
\begin{aligned}
& u_{s}=e x \\
& v_{s}=-e y .
\end{aligned}
$$

Here, $e$ is the strain rate which we have chosen to be $e=0.5$. In this case, the motion of the vortex is periodic: the vortex rotates around its centre while it remains elliptic and the aspect ratio changes periodically with time. We calculated the evolution of the vortex three times: once with the midpoint rule with 3 cycles (i.e. 4 calculations of the velocities per time step) and extrapolation (MPREXC:4), once with the fourth order Runge Kutta method (RK4) and finally with the midpoint rule with 7 cycles (i.e. 8 velocity calculations per time step) and extrapolation (MPREXC:8). In Figure 14, $\Delta A$ is plotted as a function of time $t$ for all three calculations. We see from this figure, that MPREXC:4 conserves the area of the patch better than RK4 with the same effort. Further we see that with more effort (e.g. 7 cycles), the area is even better conserved. Note that again the behaviour of the area of the patch using RK4 agrees with (12). Further, we should remark that we

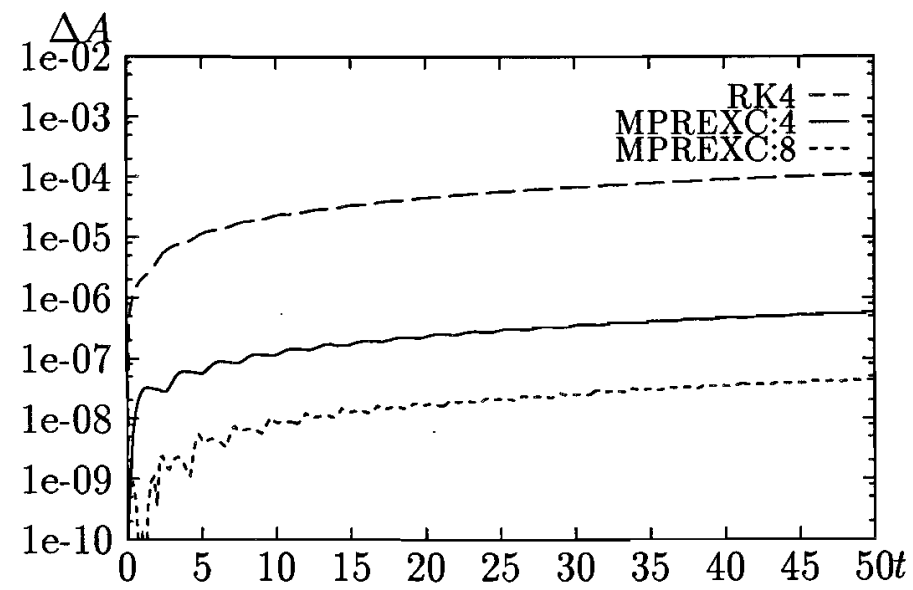

Figure 14: $\Delta A$ as a function of time $t$ for the Kida-vortex. 


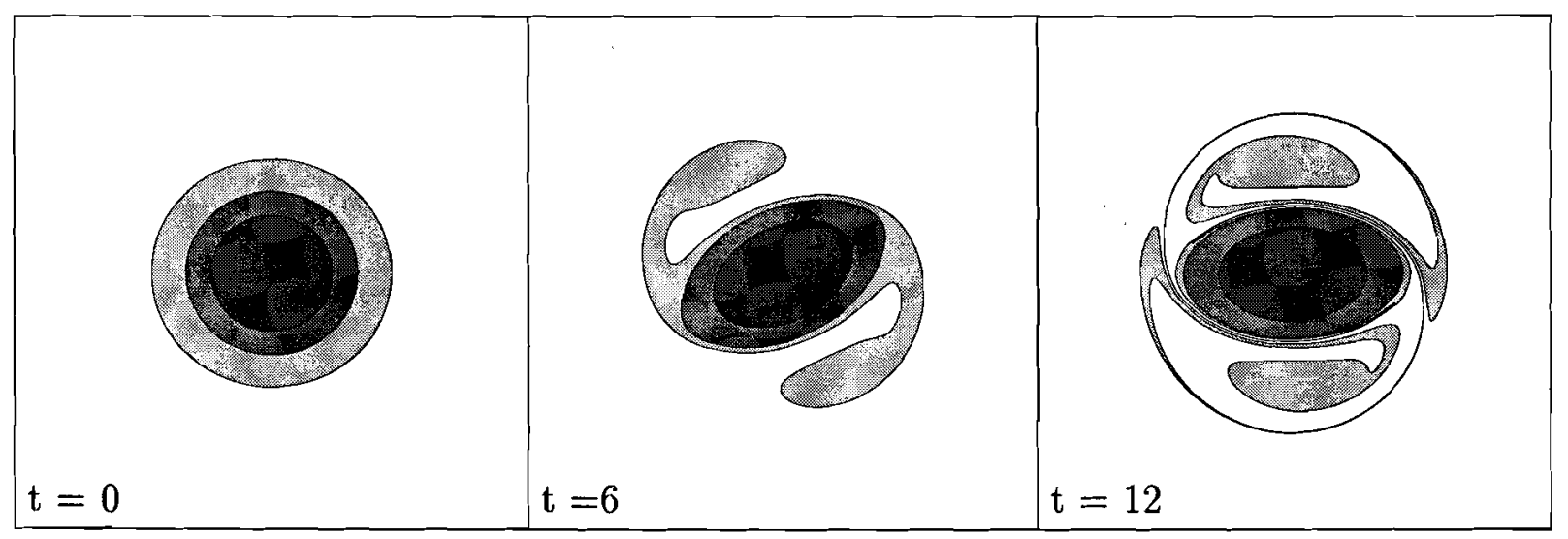

Figure 15: The evolution of a monopolar vortex into a tripolar vortex
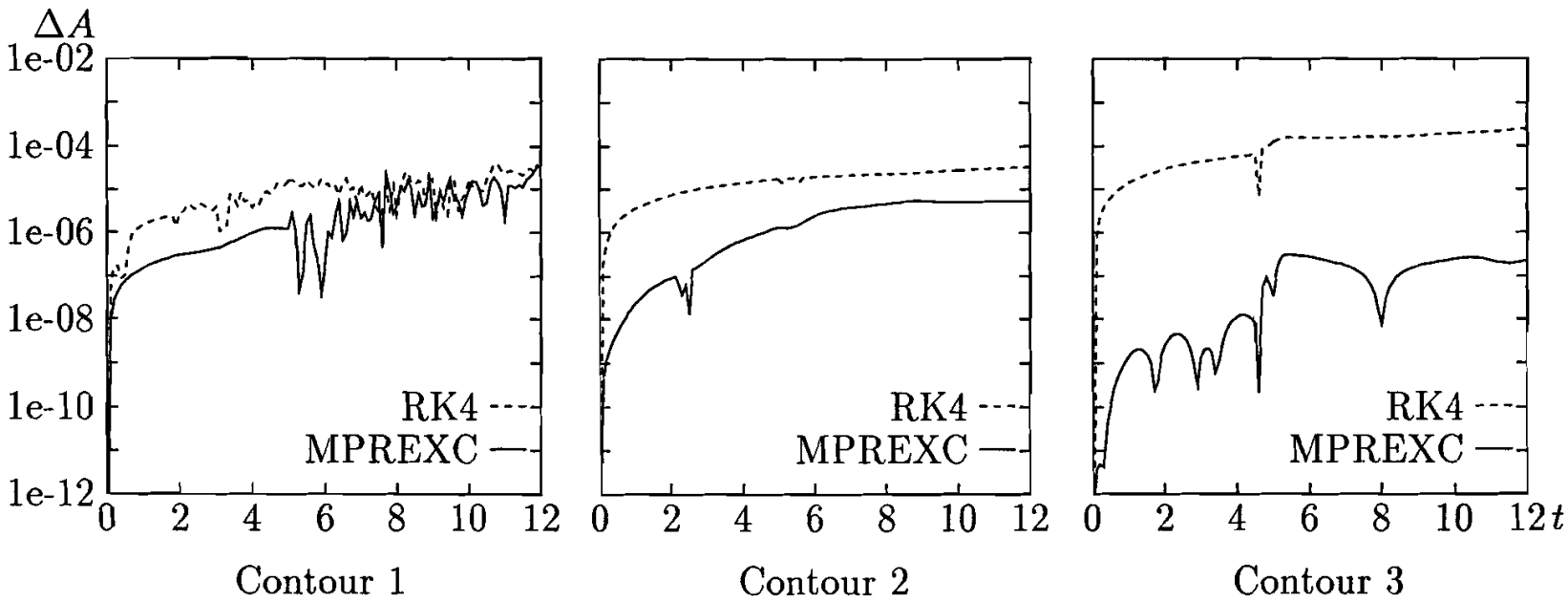

Figure 16: $\Delta A$ plotted as a function of time for contour 1 (outer contour), 2 and 3 (inner contour).

forced the number of nodes on the contour to be constant. Of course, the variation of the aspect ratio of the ellipse then causes a variation of the area, but this is the same for all calculations. If we had applied node redistribution, we would not have been sure that the effect this had on the area, would have been the same for all calculations; this would have hampered our assessment. We shall meet a similar problem with the next example.

The following example concerns the evolution of a monopolar vortex into a tripolar vortex which is a vortex consisting of an elliptic core with two satellites with vorticity of opposite sign (see e.g. [7] and [8]). The initial configuration consists of three concentric slightly elliptically disturbed contours (aspect ratio is equal to 0.95 ). The outer ring has negative vorticity, while the core (consisting of the area enclosed by the second contour) has positive vorticity. Due to the elliptical disturbance, the monopole deforms and becomes a tripole. The evolution is shown in Figure 15. As we can see from this figure, the outer 
contour deforms dramatically and this will have influence on the area enclosed by the outer contour. Although the two inner contours deform much less, nodes are added here too (in regions where the curvature became larger) which also effects the area enclosed. Since this node redistribution might be different for calculations with different time integrators (because of the growing or shrinking of the area caused by the integrator), it is hard to compare the various results. Nevertheless, we again have plotted $\Delta A$ for all three contours as a function of time both for RK4 and for MPREXC. The results are shown in Figure 16. For contour 1, both methods seem to produce comparable results from time $t=6$ on. However, this may be caused by the node redistribution since for $t<6$, MPREXC gives better results. For the other two contours, MPREXC appears to be better on the entire time interval.

\section{Conclusions}

In this paper, we have considered some aspects of the contour dynamics method. We focussed on the Hamiltonian form of the spatially discretised problem and the consequences this has for the numerical time integration. We have demonstrated that symplectic integration conserves the area enclosed by a contour better than an ordinary integration method. However, a problem with symplectic integration schemes, is that they usually are implicit. This can make implementation rather difficult. Since in general, the system of equations of the contour dynamics method is not stiff, one may use a predictor-corrector scheme for the implementation. To obtain a symplectic integrator this way, one should perform enough corrector steps, i.e. basically until machine precision is reached. However, every corrector step requires the calculation of the velocities, which is rather time consuming. Therefore, we have chosen to use an extrapolation method to accelerate the iteration process. The MPE method turns out to be very suitable for contour dynamics. It is based on the use of differences, and does not need additional information about the Jacobian matrix of the system. Performing the extrapolation for each of the contours separately, appears to work even better in practice.

Acknowledgement: We would like to thank prof. G.J.F. van Heijst who introduced us to the subject of contour dynamics.

\section{References}

[1] D.G. Dritschel. Contour surgery: A topological reconnection scheme for extended integrations using contour dynamics. J. Comput. Phys. 77:240-266, 1988.

[2] D.G. Dritschel. Contour dynamics and contour surgery: Numerical algorithms for extended, high-resolution modelling of vortex dynamics in two-dimensional, inviscid, incompressible flows. Comput. Phys. Rep. 10:77-146, 1989. 
[3] E. Hairer, S.P. Nørsett, and G. Wanner. Solving Ordinary Differential Equations 1. Springer-Verlag, 1987.

[4] E. Hairer and G. Wanner. Solving Ordinary Differential Equations 2. Springer-Verlag, 1991.

[5] S. Kida. Motion of an elliptic vortex in a uniform shear flow. J. Phys. Soc. Jpn. 50:3517-3520, October 1981.

[6] H. Lamb. Hydrodynamics. Dover Publications, sixth edition, 1932.

[7] S.J. Lin. Contour dynamics of tornado-like vortices. J. Atmos. Sci. 49:1745-1756, 1992.

[8] P. Orlandi and G.J.F. van Heijst. Numerical simulation of tripolar vortices in 2D flow. Fluid Dyn. Res. 9:179-206, 1992.

[9] J.M. Sanz-Serna and M.P. Calvo. Numerical Hamiltonian Problems. Chapman \& Hall, first edition, 1994.

[10] D.A. Smith, W.F. Ford, and A. Sidi. Extrapolation methods for vector sequences. SIAM Rev. 29:199-233, 1987.

[11] N.J. Zabusky, M.H. Hughes, and K.V. Roberts. Contour dynamics for the Euler equations in two dimensions. J. Comput. Phys. 30:96-106, 1979. 